\section{A method for the analysis of activity and spatial relations in animal groups}

\author{
MARC BEKOFF \\ Department of Environmental, Population, and \\ Organismic Biology \\ Ethology Group \\ and \\ JERRY CORCORAN \\ Computer Center \\ University of Colorado, Boulder, Colorado 80302
}

Introduction. Scientists interested in animal social organization frequently need to ask questions concerning the interindiual spatial relations among members of a group (the use of space), but often omit consideration of the ways in which interindividual spatial relations and individual activity patterns are related to one another (see Fsser, 1971, and Dodd \& Jones. 1974). The purpose of this paper is to describe a method for analyzing both the activity and spatial relations of individuals comprising a group of animals, in which the animals are visible almost all of the time.

Data collection and input. A group of 6 coyotes (Canis latrans) was observed for a period of seven months. Activity and proximity (nearness) scales and a standard score sheet. enabling the observer to record both activity and proximity at a given moment, were developed and modified after Lockwood (1975) (Table 1). The activity scale reflects changes in activity "level" on an ordinal scale, as do the proximity measures. Data were

Table 1

Activity Scale

1. Sleeping

2. Lying alert

3. Sitting

4. Standing

5. Walking (slow pace)

6. Walking (steady pace)

7. Gentle play (mouthing, etc.)

8. Excited pacing

9. Chase-play (rough-and-tumble)

Proximity (Nearness) Scalc

1. Greater than $X$ meters away

2. Greater than $Y$ meters away

3. Within $\mathrm{Y}$ meters

4. Intermittent contact (within "reach")

5. In contact collected every $10 \mathrm{~min}$ on the prepared score sheet and in this form were easily directly keypunched for computer analysis. The first 10 columns of the computer cards were used for general information (e.g., date, time of day, species, individuals), Columns 11-16 were used for activity measures (we observed a group of 6 animals), and Columns 17-31 were used to record data on dyad proximities (in our case, 15 dyads for the 6 animals). The program can handle groups of various size and can also deal with more or fewer measures of activity and/or proximity.

Questions answered. Because animals interact with one another in space and time, it is important to know the relationship between what an animal is doing (e.g., activity), how it spaces itself with respect to others, and how the other animals, in turn, space themselves with respect to a particular animal carrying out a particular act. Accordingly, three questions were raised:

(1) When a particular animal was active at a particular level, what were the activity scores (frequency) for each of the other participating or socially relevant animals? Spearman and Kendall correlation coefficients were calculated on the data: the final choice of either a Kendall or Spearman analysis depended on the nature of the data (Siegel, 1956).

(2) When the proximity score for a particular dyad was known, what were the activity scores (frequency) for each of the members of the dyad? Or, what did each of the animals do when they were a particular distance from one another?

(3) When the proximity score for a dyad was known, what was the distribution of proximity scores for all of the other dyads? As with Question 1, correlation coefficients were calculated.

Programming notes. Four separate programs were custom written to handle the data with the input and output formats predetermined. All analyses were performed on a CDC- 6400 computer using the Kronos 2.0 operating system. These programs are adapted to handle other data on a case-by-case basis. The user can specify the format of his input and any labeling desired on the output. The data sheet used, as well as all programs, are available at no cost from the senior author, and are referred to as the CANID FILE. Contact Marc Bekoff, Dept. of Environmental, Population, and Organismic Biology, University of Colorado, Boulder, Colorado 80302.

\section{REFERENCES}

Dodd, W. P. \& Jones, E. A program for the analysis of intragroup interaction based on spatial dynamics. Behavior Research Methods and Instrumentation, 1974, 6, 497-503.

Esser, A. H. (Ed.) Behavior and environment: The use of space by animals and man. Plenum Press, 1971. P. 411.

Lockwood, R. Cohesive and divisive social factors in captive wolves: A dyadic and pack analysis. $\mathrm{Ph}$. D. dissertation, $\mathrm{St}$. Louis, Washington University, 1975 .

Siegel, S. Non-parametric statistics, McGraw-Hill, 1956. P. 312. 